\title{
Concentrate for Dip Emulsion Dosage
} Form

National Cancer Institute

\section{Source}

National Cancer Institute. Concentrate for Dip Emulsion Dosage Form. NCI Thesaurus.

Code C149386.

Liquid preparation intended to be diluted in the specified liquid to obtain a dip emulsion. 